\title{
X-Ray Diffraction Effects Given by the Austenitic Phase During the Training Process of Shape Memory Cu-Zn-Al Polycrystals
}

\author{
C. Jourdan, G. Guénin*, S. Belkahla**, J. Gastaldi and G. Grange \\ CRMC2-CNRS, Campus de Luminy, Case 913, 13288 Marseille cedex 09, France \\ * INSA-G.E.M.P.P.M., 20 avenue Albert Einstein, 69621 Villeurbanne cedex, France \\ ** Université Annaba, BP. 12, 23000 Annaba, Algérie
}

\begin{abstract}
To visualize the grain boundary role on the shape memory property of $\mathrm{Cu}-\mathrm{Zn}-\mathrm{Al}$ alloy, we have followed the austenitic structure evolution, of crystals submitted to a training treatment, by synchrotron X-ray topography in white beam. Single crystals, tricrystals and polycrystalline samples have been studied. The comparison of results shows that in polycrystalline samples large, non recoverable stresses, develop in all grains. These stresses must be taken into account when the origin of the shape memory property is discussed.
\end{abstract}

\section{INTRODUCTION}

In the last forteen years the shape memory property and associated phenomena : pseudoelasticity, two-way memory effect (T.W.M.E.) ... have been well investigated and have given some industrial and domestic applications. Difficulties arise when it is looked into the origin of T.W.M.E.

Schematically this effect results from the selection of one (or two) transformation variant(s) by the training process and from the concomitant development of oriented stress centers well distributed in the crystal. It is supposed that these ones favour the nucleation, the growth and the backward movement of the trained transformation variant when the training stress is suppressed. Today the role of the training process in the creation or the activation of these stress centers is not well understood.

In precipitate free single crystals these centers should be the residual stress fields associated with the dislocation arrays, experimentally observed and characterized, resulting from the training process [1]. After transformation cycling the problem of the variant selection among 24 crystallographically equivalent variants has been discussed by Sade et al [2], Lovey et al. [3] and Pons et al. [4]. The energy contribution of an isolated dislocation in the austenitic structure has been evaluated and with hypothesis on Burgers vectors and interaction energy between dislocations in a same area it is possible to approach the selection mechanism. A quite similar conclusion has been suggested by Stalmans et al. [5] who have shown that the crystallographically equivalent transformation variants are not thermodynamically equivalent. If single crystals contain precipitates Lovey et al. [6] have shown that during the training process dislocations are generated by these precipitates and this dislocation-precipitates association develops internal stresses in the direction which should favour the trained transformation variant. The role of the local martensite plates retained above $A_{f}[7]$ on the TWME origin is not preponderant [8]. This survey from some main studies shows that the TWME in single crystals is closely dependent on the training dislocations. The case of polycrystals seems different. It is generally admitted and verified that the training of a polycrystal is easier than the training of single crystal. Several thousands of pseudo-elastic cycles are necessary to obtain a good TWME in $\mathrm{Cu}-\mathrm{Zn}$-Al single crystals when some tens are sufficient for polycrystals. A first explanation will be to consider the grain boundaries acting as nucleation centers (dislocations or precipitates). In fact the problem is more intricate. In single crystals whatever was the training method the stress applied selects one (or two) transformation variant(s). So, the structure of the austenitic or martensitic phases appears macroscopically homogeneous as well in orientation as in dislocation density.

A polycrystal in austenitic phase consists of great number of grains with various orientations. On cooling, during the training, these grains transform but they are not free to transform as they were lonely. In contrast to the case of single crystals, where the transformation variant selected by the applied stress satisfies a criterion similar to the "Schmidt" criterion (S) in crystal plasticity, in polycrystals each grain will try to accommodate the inhomogeneous strain field due to neighbouring crystals by developing several 
types of variants : variants whose orientation is close to the orientation of variants predicted by the application of the "Schmidt" criterion and "secondary" variants localised at the grain boundary vicinity, to accomodate the deformation between grains. The large change in symmetry during the cubic to orthorhombic transformation authorizes this accommodating effect [9]. The non self-accommodating character of these different variants must be pointed out. The thinner the polycrystal will be the more this effect will be marked.

The influence of grain boundaries on the martensitic transformation in polycrystals has been recently discussed through symmetry relations between the two phases by Bhattacharya and $\mathrm{Kohn}$ [9]. In $\mathrm{Cu}-\mathrm{Zn}-\mathrm{Al}$ alloys there are few works imaging the structure evolution on polycrystals compared to studies on single crystals. The thermodynamic and the mechanisms of the TWME has been studied by Stalmans et al [8]. Guilemany et Gil [10] have shown the role of the grain boundaries in the shift of the $\mathrm{M}_{S}, \mathrm{M}_{\mathrm{f}}, \mathrm{A}_{\mathrm{S}}$ and $\mathrm{A}_{\mathrm{f}}$ temperatures [10]. To our knowledge, except the work of Delaey et al. [11], by optical microscopy, there is no direct evidence of the grain boundary role on the structure of the polycrystals in the austenitic phase during and after the training process.

The good TWME in polycrystals was frequently assigned to stress concentrations at the vicinity of grain boundaries [13]. We have tried to visualize these stresses by synchrotron $X$-ray topography which is the well adapted experimental method to display the long range stresses in crystals. The structure evolution of single, tri and polycrystals have been followed during the training process and the structure of austenitic phases have been compared.

\section{EXPERIMENTAL TECHNIQUE}

Detailed information on the technique used in this study is available in [2]. Single and tri-crystals were cut from an ingot obtained by a modified Bridgman technique while polycrystalline samples are cut from an annealed sheet whose grain diameter are close to $8.10^{-4} \mathrm{~m}$. After cutting the crystals were mechanically and electrolytically polished. Their $M_{S}$ point, when the stress is applied, is close to $263 \mathrm{~K}$. The cross section of the incident beam is limited to the size of the sample so diffraction patterns give information on the structure of the whole crystals. Experiments were made in a Peltier cell fitted with a tensile machine. An uniaxial stress close to $4 \mathrm{MPa}$ is applied to the crystals during the training. The temperature cycling is in the range 243-303 K. The time exposure to record diffracted beams on films is $1-5$ seconds. The originality of the experimental method used resides in the high flux and the low divergence of the $\mathrm{X}$-ray synchrotron beam which allows to follow in situ the crystal structure evolutions.

Generally the $B$ and $9 R$ terms are used for simplicity, instead of $\mathrm{DO}_{3}$ and $18 \mathrm{R}$, to indicate the high and low temperature phases having respectively b.c.c. and $9 \mathrm{R}$ structures.

\section{EXPERIMENTAL RESULTS}

\section{- Single crystals}

Figure 1 shows the topogram given by a single crystal in $B$ phase before training.

Figure 2 shows the topogram given by the same single crystal in $B$ phase after 14 thermal cycles with a training stress applied of $4 \mathrm{MPa}$.

It is worthy to note that the diffraction spot after 14 cycles is rectangular and its shape is about the same as the one given by the initial state. Inside the topograms linear contrasts are clearly visible. They correspond to the dislocation arrays described by Malaria et al [1]. Their traces are parallel to the intersection of the habit plane with the crystal surface. From these traces it is possible to determine the orientation of the transformation variants [14]. It has been shown that systematically in single crystals these variants obey to the "Schmidt" criterion and that generally a single crystal in B phase gives a single crystal in $9 R$ phase [15]. When the number of cycles increases the diffraction spot remains rectangular but the number of dislocations in arrays increases so that the linear contrasts become blurred. Compare the arrays in figure 3 given by a single crystal which has been submitted to 400 training cycles to a detail of an array after 1 training cycle ( figure 4). The dislocations constituting this array are well resolved.

\section{- tricrystal}

Figure 5 - Structure of the tricrystal in austenitic phase before training. the three topograms are put side by side in the crystal configuration.

Figure 6 - Same topograms given by the tricrystal after 12 training cycles

Note the influence of grain boundaries on the B-9R transformation. The aftermaths of the $9 R$ phase in grain 1 are localized at the 1-3 boundary, in grain 2 two directions of dislocation arrays are detected : 


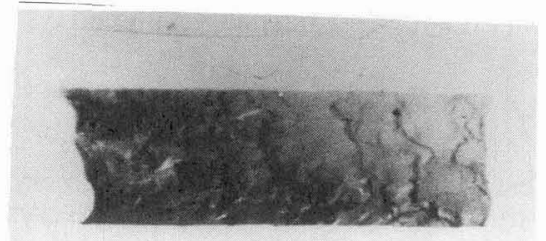

Figure 1 : Initial state 112 reflexion. $\beta$ phase

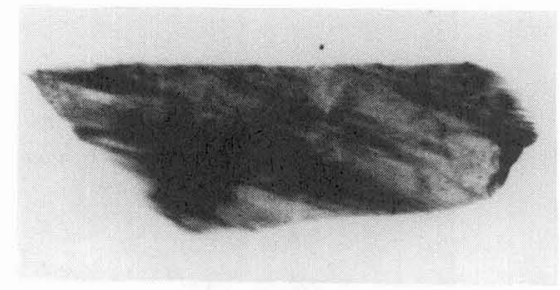

Figure $3:$ Topogram given by a single crystal after 400 cycles. $\beta$ phase

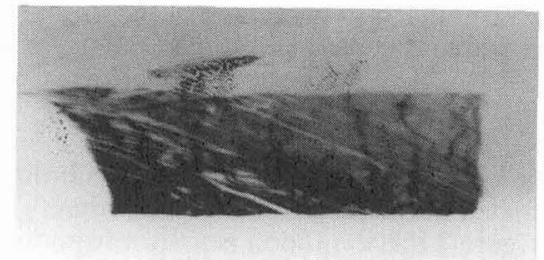

Figure 2: After 14 thermal cycles. $\beta$ phase

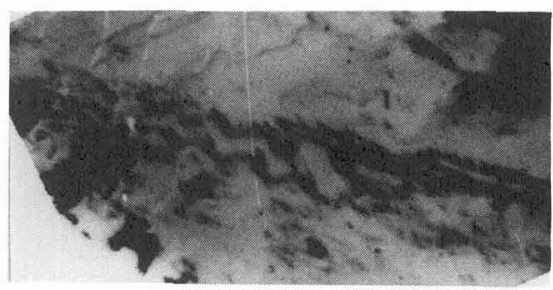

Figure 4 : Detail of a dislocation array after 1 cycle. $\beta$ phase

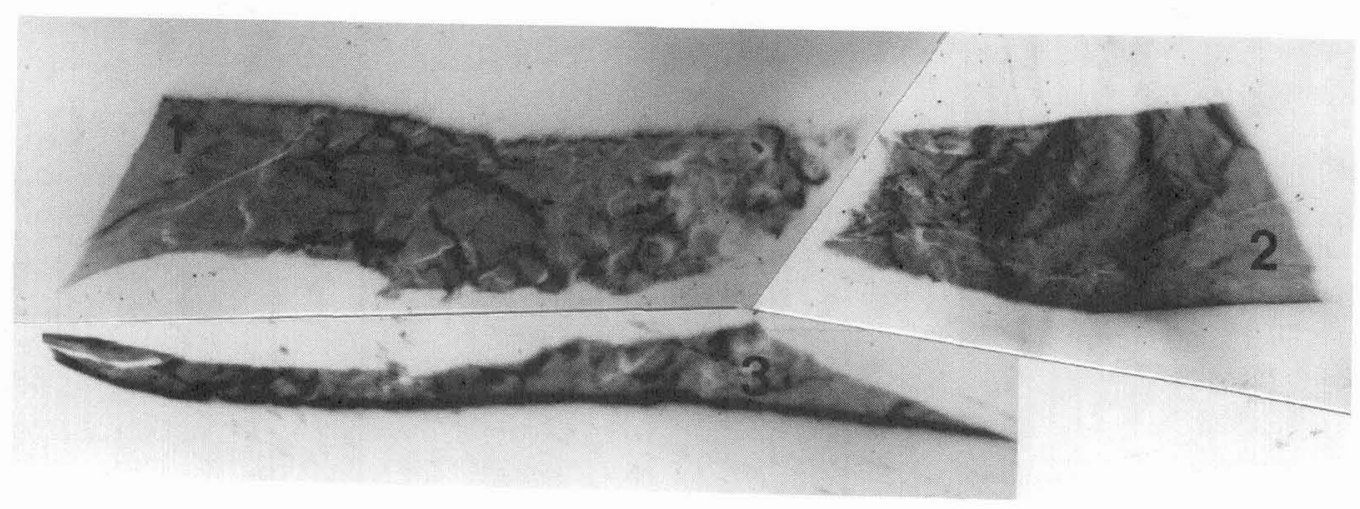

Figure 5 : Tricrystal before training. $\beta$ phase

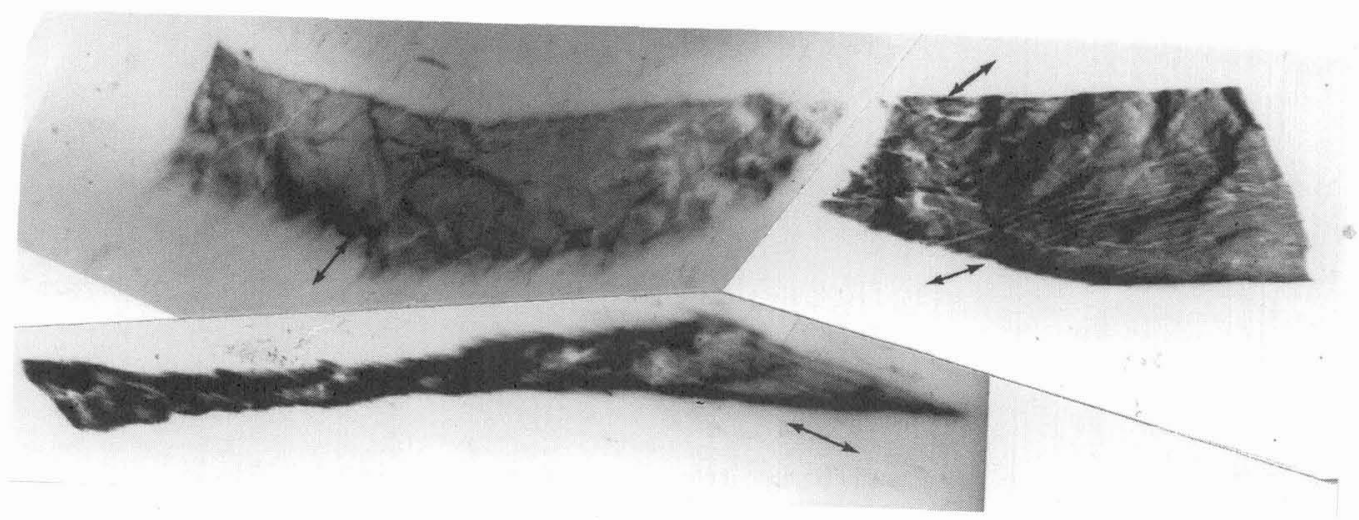

Figure 6: Same tricrystal as figure 5 after 12 training cycles 
dislocation arrays generated at the 2-3 boundary and arrays localised at the 2-1 boundary. In grain 3 these arrays are numerous and spread out in the whole crystal.

\section{- Polycrystal}

When a polycrystalline sample is submitted to a training process the structure evolution of each crystal included in the matrix, taken individually, is different from the structure evolution of a large single crystal. In figure 7 this evolution is shown from two grains representative of the polycrystalline matrix evolution.

The role of grain boundaries is displayed in these topograms. Two main experimental results are evidenced: as early as the first cycles the small crystals in austenitic phase are submitted to large internal stresses visualized by the elongated shape of the diffraction spots and the well organised substructure observed in large single crystals with parallel dislocation arrays is not visible.
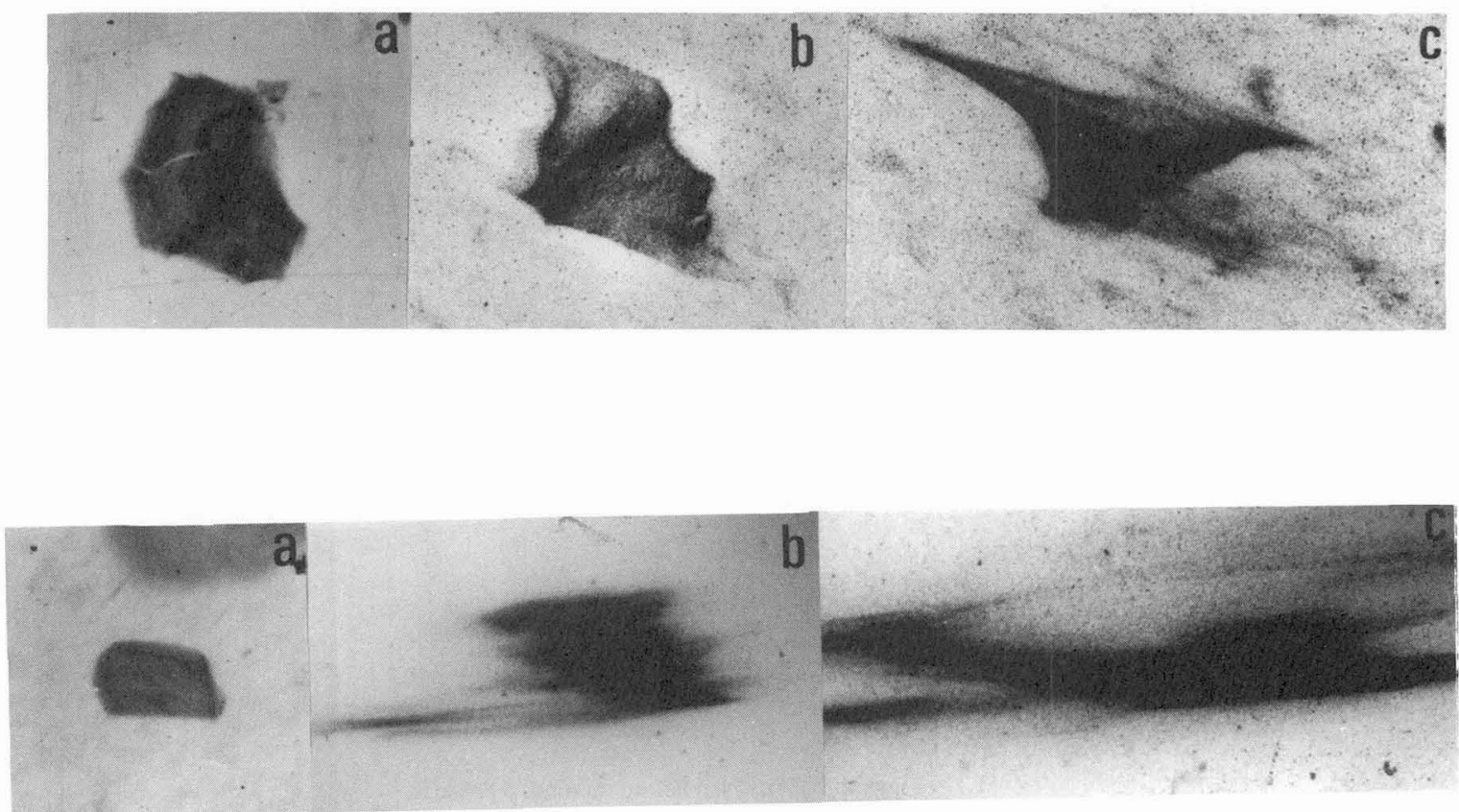

Figure 7 : Structure evolution of two grains in $\beta$ phase include in the polycrystalline matrix. $\mathrm{a}$ : initial state, $\mathrm{b}$ : two training cycles, $\mathrm{c}$ : five training cycles. 


\section{DISCUSSION}

In large single crystals, experimental results confirm the electron microscopic observations. We show that the dislocations, in arrays, are generated from the outset cycling. Their number increases with the number of training cycles and their crystallographic orientation and density are comparable to those observed by electron microscopy [1]. The rectangular shape of the diffraction spots even after few hundred cycles indicates that the dislocations induced in the crystal by the training process do not induce macroscopic rotation of the crystal net. It seems probable that the shape memory property in single crystals results from these dislocations arrays which are the sole crystalline defects observed after training.

In polycrystals with large grains illustrated by the tricrystal in our study the structure evolution of the austenitic phase depends on the relative orientations of these grains. When a grain is well oriented with regard to the applied stress, dislocation arrays are observed and the trained variant is in accordance with the "Schmidt" criterion. See for example the grain 3 in figure $6 . S \simeq 0.984$. The maximum value of $S$ is taken equal to 1 . The boundary effects are visible in grain 1 and 2 . In grain 1 the $S$ criterion of the transformation variant which has appeared is near by 0.827 when the variant whose $S \simeq 0.994$ should be observed. In a same manner in grain 2 the variants developed have respectively a $S \simeq 0.984$ (2-3 boundary) and $S \simeq$ 0.914 (2-1 boundary) when the $S$ maximum is 0.995 . These two arrays are generated at the crystal boundary. After 12 training cycles a slight undulation which intensifies with the number of cycles is detected. The fundamental observation displayed in the polycrystalline austenitic phase evolution is the development of large stresses during the training process. The diffraction spots are undulated and stretched. This elastic deformation of the austenitic crystals is not recoverable and persists even if the training stress applied is suppressed. The whole crystal is affected and the dislocation arrays are invisible. In polycrystals with fine grains, owing to the boundary influences the martensitic transformation is not monovariant. The dislocation arrays which are linked to the habit planes can take several directions. So they are much less organised than in single crystals. On the other hand the structure inhomogeneities resulting from the polycrystal transformation introduce certainly dislocations different from the interphase dislocations. These two remarks can explain why in polycrystals the dislocation arrays are not detected. This is the fundamental point evidenced by the $\mathrm{X}$-ray diffraction diagrams. Owing to the limited place in this paper, the martensitic phase structure evolution has not been described. Briefly the grain boundaries influence also this structure. Large single crystals in austenitic phase transform in large single crystals in martensitic phase which grow by adjunction of martensitic plates belonging to the same transformation variant [15]. In polycrystals this growth process cannot be observed. Owing to the various orientations all grains do not transform in the same time and the growth of some martensitic grains included in the austenitic polycrystalline matrix induces large stresses in the sample which do not act necessarily in the direction of the stress applied. The final polycrystalline martensitic matrix, except some crystals, is highly strained, even when the training stress is suppressed.

\section{CONCLUSION}

The sensitivity of the X-ray diffraction methods to the deformation of lattice planes of crystals has been used in this study to visualize the grain boundary influences during the martensitic transformation of $\mathrm{Cu}$ $\mathrm{Zn}-\mathrm{Al}$ polycrystalline shape memory alloy submitted to a training process. The dislocation arrays which appear and develop during the training treatment are not the preponderant effect in polycrystals. The mutual interaction between grains of different orientations develops, during the cycling, non recoverable large stresses in whole crystals. These stresses, contrary to the hypothesis generally expressed, are not localised at or in the grain boundary vicinity. Whole crystals are affected and this effect is more important for polycrystals with fine grains. These stresses are certainly responsible for the better shape memory property of polycrystals and must be taken into account when the origin of the shape memory property is discussed.

\section{References}

[1] Malaria J., Sade M. and Lovey F.C., J. Phys. IV - Colloque C8 - ICOMAT 95 (Editions de Physique 1995), pp. 889-894

[2] Sade M., Hazarabedian A., Uribarri A. and Lovey F.C.; Proc. Int. Conf. on Solid Phase Transformations (Edited by G.W. Lorrimer, 1988) pp. 279-280

[3] Lovey F.C., Hazarabedian A. and Garces J., Acta Metall. Mater. 37 (1989) pp. 2321-2327

[4] Pons J., Lovey F.C. and Cesari E., Acta Metall. Mater. 38 (1990) pp. 2733-2740

[5] Stalmans R., Van Humbeck J. and Delaey L., Acta Metall. 40 (1992) 2921-2931 
[6] Lovey F.C., Torra V., Isague A., Roqueta D. and Sade M., Acta Metall. Mater. 42 (1994) pp 453-460

[7] Perkins F. and Sponholz., Metall. Trans. 15A (1984) pp. 313-321

[8] Stalmans R., Van Humbeck J. and Delaey L., Acta Metall. Mater. 40 (1992) pp. 501-511

[9] Bhattacharya K. and Kohn R.V., Acta Metall. Mater. 44 (1996) pp. 529-542

[10] Guilemany J.M. and Gil F.J., Mater. Res. Bull. 25 (1990) pp. 1325-1332

[11] Delaey L., Van de Voorde F. and Krishnan R.V., Shape Memory Effects in Alloys (International Symposium, Toronto 1975), pp. 351-365

[12] Jourdan C., Grange G., Gastaldi J., Roques V., Belkahla S. and Guenin G., Acta Metall. Mater. 43 (1995) pp. 4213-4225

[13] Guenin G., J. Phys. IV - Colloque C2 - ESOMAT 94 (Les Editions de Physique 1994), pp. 325-334

[14] Jourdan C., Gastaldi J., Grange G., Belkahla S. and Guenin G., Acta Metall. Mater. 43 (1995), pp. $4227-4234$

[15] Jourdan C., Gastaldi J., Grange G. and Guenin G., J. Phys. IV - Colloque C8 - ICOMAT 95 (Editions de Physique - 1995) pp. 859-863 